\title{
Gesetzgeberische Eingriffe in die Privatautonomie und ihre Folgen. Das Beispiel der Sicherungsrechte im Mietvertrag
}

\section{Einleitung \\ 1. Das Sicherungsbedürfnis im gegenseitigen Vertrag}

Wer einen Vertrag schließt, kennt seinen Partner oft nur oberflächlich. In den meisten Fällen ist das unproblematisch. Zahlreiche Geschäfte des täglichen Lebens werden unmittelbar nach dem Vertragsschluß erfüllt, und vor Unsicherheiten im Hinblick auf die Leistungsfähigkeit der Gegenseite schützt die Erfüllung Zum um Zug (§§ 320, 322 BGB). Das gilt auch bei Verträgen über wertvolle Kaufgegenstände. Bei Immobiliengeschäften kann sich der Verkäufer durch Grundschulden oder Hypotheken absichern und geht nur ein geringes wirtschaftliches Risiko ein. Dasselbe gilt beim Autokauf, wenn der Käufer den Kredit einer mit dem Händler verbundenen Bank in Anspruch nimmt und dieser sein Fahrzeug zur Sicherheit übereignet. Die Sicherungsrechte zugunsten des Verkäufers ermöglichen dem Käufer die Teilnahme am Wirtschaftsleben, auch wenn seine finanziellen Spielräume begrenzt sind. Die Vereinbarung einer Sicherheit kommt also auch dem wenig zahlungskräftigen Verbraucher zugute. Die inhaltliche Gestaltungsfreiheit von Schuldverträgen bindet sozial schwächere Bevölkerungskreise in den Geschäftsverkehr ein. ${ }^{1}$

Bei Verträgen, deren Erfüllung längere Zeit beansprucht, kann sich das Sicherungsinteresse verschieben. Bei termingebundenen Bauarbeiten wird häufig eine Vertragsstrafe vereinbart, falls der Bauunternehmer mit der Fertigstellung in Verzug gerät. Der Bauherr läßt sich die Sicherheit hier nicht wegen einer unsicheren wirtschaftlichen Lage des Unternehmers gewähren, sondern benutzt sie ausschließlich als Druckmittel zur ordnungsgemäßen Vertragserfüllung.

Wiederum anders ist die Interessenlage bei Mietverträgen beschaffen. Dem vergleichsweise hohen Wert der vermieteten Sache steht ein Mietzins gegenüber, der lediglich den Gebrauchsvorteil, nicht aber den Sachwert abdeckt. ${ }^{2}$ Außerdem kann es ungewiß sein, ob der Mieter die Miete pünktlich und in voller Höhe bezahlt. Auf der anderen Seite ist es für den Mieter ungewiß, ob der Vermieter seine Instandhaltungsund Gewährleistungspflichten ordnungsgemäß erfüllt und Nebenkostenabrechnungen pünktlich und nachvollziehbar erstellt. Wegen der vergleichsweise langen Laufzeit

1 Adams, Ökonomische Analyse der Sicherungsrechte, 1980, 271.

2 Die Gesetzesbegründung zur Mietrechtsreform 2001 spricht davon, der Vermieter müsse sein Eigentum "angemessen wirtschaftlich verwerten" können: BT-Drucksache 14/4553, hier zitiert nach dem Abdruck bei Grundmann, Mietrechtsreformgesetz. Einführung Neues Recht - Materialien, 2001, 71. 
insbesondere von Wohnungsmietverträgen können Vertragsstörungen und Probleme auftauchen, die bei eher punktuellen rechtsgeschäftlichen Kontakten wie Kaufverträgen nicht auftreten. Das Sicherungsinteresse der Parteien ist also darauf gerichtet, Vertragsstörungen nach Möglichkeit zu vermeiden und Pflichtverletzungen unkompliziert zu ahnden, ohne kündigen oder klagen zu müssen.

Mit dieser Situation hat sich die neue Institutionenökonomik eingehend beschäftigt. Sie wendet das aus der Unternehmenstheorie bekannte Prinzipal-Agent-Modell auf Dauerschuldverhältnisse an, die durch unterschiedliche Informationen, Interessen und Gefährdungslagen der Parteien gekennzeichnet sind. Danach entstehen typische Überwachungsprobleme, wenn in einem Vertrags- oder Hierarchieverhältnis der eine Teil dafür verantwortlich ist, das Vermögen des Partners zu schützen oder dessen Rechtsgüter zu schonen. Der sachnähere Agent verfügt über Sonderwissen und verfolgt zudem seine persönlichen Interessen, die der Prinzipal oftmals nicht kennt. Diese Gefahr bezeichnet man als moralisches Risiko. ${ }^{3}$ Demgegenüber muß der Prinzipal Zeit, teilweise auch Geld aufwenden, um die ordnungsgemäße Vertragserfüllung des Agenten zu überwachen. Die Informationsasymmetrie verursacht somit Transaktionskosten, die dazu führen können, daß bestimmte Vertragsgestaltungen wirtschaftlich unattraktiv werden. ${ }^{4}$ Da der Prinzipal, also etwa der Vermieter, den Agenten, also den Mieter, beim Vertragsschluß zumeist nicht näher kennt, ist das Ausmaß des moralischen Risikos nur schwer abzuschätzen. Der Prinzipal kann seine Überwachungskosten aber dadurch minimieren, daß er sich vom Agenten eine Sicherheit gewähren läßt, die man, wiederum ökonomisch gesprochen, als Geisel bezeichnet. ${ }^{5}$ Weil der Agent nunmehr ein eigenes Interesse daran hat, die von ihm gestellte Sicherheit zurückzuerlangen, hat er nach der Lehre vom Rationalverhalten ${ }^{6}$ einen wirtschaftlichen Anreiz, bei der Erfüllung seiner Pflichten die Interessen des Prinzipals zu verfolgen. Je höher die gestellte Sicherheit, um so geringer ist die Gefahr, daß sich das vom Agenten ausgehende moralische Risiko realisiert. Der Extremfall einer solchen Vertragsgestaltung wäre ein Kauf mit Rückkaufoption zum jeweiligen Zeitwert oder eine Sicherheit in derselben Höhe. ${ }^{7}$ Dies ist dem Agenten typischerweise aber wirtschaftlich nicht möglich, sonst würde etwa ein Mieter sofort Wohneigentum erwerben. Der andere Extremfall wäre ein vollständiger Verzicht auf eine Sicherheit bei einer besonders ausgeprägten Gutgläubigkeit des Prinzipals oder einer engen emotionalen Bindung der Parteien. Zwischen diesen beiden Polen werden die Parteien im Normalfall eine Vereinbarung treffen, deren genauer Inhalt von mehreren Variablen abhängt. Beim Mietvertrag sind dies die Vertragsdauer, die Miethöhe sowie typische Risikofaktoren in der

3 Richter, Institutionen ökonomisch analysiert, 1994, 16-17; zu dieser Lehre auch Kirchner, Ökonomische Theorie des Rechts, 1997, 13.

4 Richter/Furubotn, Neue Institutionenökonomik, 2. Aufl. 1999, 163-171.

5 Richter/Furubotn (wie Anm. 4), 175-176.

6 Hierzu Kirchner (wie Anm. 3), 13-18.

7 Richter/Furubotn (wie Anm. 4), 97, am Beispiel des Nießbrauchs; ähnlich Eger, Eine ökonomische Analyse von Langzeitverträgen, 1995, 178-179, wonach eine ideale Sicherheit beim Kaufvertrag dem positiven Interesse des Verkäufers an der Vertragsdurchführung entspricht. 
Person des Mieters. In diesen Punkten unterscheiden sich in der Theorie sämtliche Verträge voneinander. Die Verschiedenartigkeit der Lebenssachverhalte führt in einem auf der Privatautonomie beruhenden Zivilrecht zu keinen ernsthaften Regelungsproblemen. Je nach Interessenlage können die Parteien vom gesetzlichen Leitbild abweichen und Vertragsstrafen und Sicherheitsleistungen vereinbaren und auch die Erfüllung Zug um Zug abbedingen.

\section{Die Einschränkung der Vertragsfreiheit durch einseitig zwingendes Privatrecht}

Das soeben skizzierte Modell läßt sich nur mit erheblichen Einschränkungen im Wohnungsmietrecht wiederfinden. Als weiterer Faktor, der bei den konkreten Vertragsgestaltungen $\mathrm{zu}$ berücksichtigen ist, erlangen nämlich die einseitig zwingenden Mietrechtsvorschriften zentrale Bedeutung. Die individuelle Vertragsgestaltung für die jeweils individuellen Bedürfnisse der Vertragsparteien ist vom Gesetzgeber aus Gründen des Mieterschutzes eingeschränkt worden. Die Umwandlung des Mietrechts vom dispositiven Privatrecht zu einem weitgehend einseitig zwingenden Recht erfolgte zunächst unter dem Vorzeichen der Wohnungsnot. In Zeiten der Wohnraumknappheit, so lautete die Überlegung des Gesetzgebers, könne der Wohnungssuchende seine Freiheit nicht aktiv wahrnehmen, sondern sei gezwungen, auf die vom Vermieter gestellten Bedingungen einzugehen. Die Einschränkung der Vertragsfreiheit verfolgte den Zweck, eine annähernd gleiche Verhandlungsposition herzustellen. Als politisches Ziel stand die Versorgung der Bevölkerung mit bezahlbarem Wohnraum im Hintergrund. $^{8}$

Seit den sechziger Jahren kann von einer existentiellen Wohnungsnot kaum noch die Rede sein. Die Einschränkung der Vertragsfreiheit im Mietrecht wurde dennoch seit den siebziger Jahren immer stärker ausgebaut. Der Mieterschutz und damit die Eingriffe in das Eigentum wurden aber nun anders begründet. Nicht mehr die Wohnungsknappheit diente als Argument, sondern die überragende Bedeutung der Wohnung als Mittelpunkt der menschlichen Existenz. ${ }^{9}$ Nach der Mietrechtsreform vom September 2001 ist die Abkehr vom dispositiven Privatrecht nunmehr soweit vorangeschritten, daß mehrfach ausdrückliche Erlaubnisnormen in den Gesetzestext aufgenommen wurden, um abdingbare Normen besonders kenntlich zu machen ( $\S 5551$ Abs. 3 S. 2, 556 Abs.1, 557 Abs. 1 und 2, 557 a Abs. 1, 557 b Abs. 1 BGB). ${ }^{10}$

Das Mietrecht eignet sich somit besonders gut, um den Einfluß staatlicher Gesetzgebung auf die Vertragsgestaltung zu untersuchen. Hierbei sollen die Sicherungsrechte

8 Guter historischer Überblick bei Honsell, Privatautonomie und Wohnungsmiete, in: AcP 186 (1986), 115-186 (119-122); Staudinger/Emmerich, Kommentar zum Bürgerlichen Gesetzbuch, 13. A. 1995, vor $\S \S 535,536$ Rn. 13-29; aus zeitgeschichtlicher Perspektive Lutz, Der Mieterschutz der Nachkriegszeit. Einfluß des Mietrechts auf den Wohnungsbau, 1998.

9 So bereits das BVerfG NJW 1964, 1848 (1850); hierzu Honsell (wie Anm. 8), 117, 123, 139; Emmerich/Sonnenschein, Miete. Handkommentar, 7. A. 1999, vor §§ 535, 536 Rn. 36 (Emmerich).

10 Hierzu Oestmann, Erlaubnisnormen im Mietrecht - ein Prinzipienbruch?, in: NZM 2003, $1-6$. 
im Mittelpunkt der Überlegungen stehen. Wegen der mieterschützenden Zielrichtung der zwingenden Gesetzesnormen beschäftigt sich diese Untersuchung ausschließlich mit den Sicherungsmöglichkeiten des Vermieters. Die Zurückbehaltungsrechte des Mieters, seine Aufrechnung oder Hinterlegung werden im folgenden nicht problematisiert. Da die mietrechtlichen Vorschriften insoweit dispositiv sind, ${ }^{11}$ läßt sich der Einfluß zwingender Privatrechtsnormen auf die Vertragsgestaltung nur am Beispiel der Sicherungsrechte zugunsten des Vermieters aufzeigen. Die einseitige Gewichtung der Ausführungen beruht demnach auf der vom Gesetzgeber geschaffenen Rechtslage.

Der unabdingbare Ausschluß bestimmter Sicherungsmöglichkeiten des Vermieters sowie eine Begrenzung der zulässigen Sicherungshöhe führt zu einer erheblichen Veränderung der Vertragsgestaltung im Vergleich zu einem privatautonom vereinbarten Vertrag. Das von der Prinzipal-Agent-Theorie angenommene Modell bedarf somit erheblicher Differenzierungen. Ziel der folgenden Untersuchung ist es zu prüfen, ob die vom Gesetzgeber beabsichtigte Besserstellung des Mieters durch die Einschränkung der Sicherungsmöglichkeiten des Vermieters den gewünschten Erfolg hatte und welche weiteren Folgen sich hieraus ergeben. Der Blick fällt somit auf die tatsächlichen und ökonomischen Folgen der zwingenden Gesetzesvorschriften.

Zunächst soll die aktuelle Rechtslage dargestellt werden. Sodann werden die Auswirkungen der unflexiblen Sicherungsmöglichkeiten auf das Verhalten der Parteien beschrieben. Ausgehend von diesen Ergebnissen kann sodann der Eingriff des Gesetzgebers in die Privatautonomie grundsätzlich problematisiert und erörtert werden. Hierbei wird sich zeigen, daß man einen ohne staatliche Lenkung entstandenen Bereich des Rechts- und Geschäftsverkehrs durch gezielte Einzeleingriffe nur bedingt planen kann. Die hoheitlichen Eingriffe mögen zwar punktuell das vom Gesetzgeber verfolgte Ziel erreichen, verursachen aber zumeist unbeabsichtigte Nebenwirkungen, die dem Zweck der Regelung entgegenlaufen. In Zweifelsfällen empfiehlt es sich daher, die Privatautonomie nicht einzuschränken, sondern auf ihre Regelungsmechanismen zu vertrauen. Das ist nicht nur angesichts der geringen Planbarkeit komplexer Ordnungen zweckmäßig, sondern entspricht der traditionellen Konzeption des Privatrechts schlechthin. Überdies gebietet die Verfassung die umfassende Gewährleistung der allgemeinen Handlungsfreiheit (Art. 2 Abs. 1 GG).

\section{Die einzelnen Sicherungsmöglichkeiten im Mietvertrag}

In Deutschland wohnen 60-70 \% der Bevölkerung in Mietwohnungen. ${ }^{12}$ Etwa 50 Millionen Mietern und ihren Angehörigen stehen rund 10 Millionen Vermieter

11 Selbst $\$ 570$ BGB, der dem Mieter ein Zurückbehaltungsrecht gegenüber dem Herausgabeanspruch des Vermieters versagt, ist abdingbar, so bereits die Rechtsprechung zum früheren $§ 556$ Abs. 2 BGB, zustimmend Palandt/Weidenkaff, Bürgerliches Gesetzbuch, 61. A. 2002, § 570. n. F. Rn. 2.

12 Das ist der höchste Wert in Westeuropa: Vergleichszahlen aus den achtziger Jahren bietet der Kronberger Kreis, Mehr Mut zum Markt, 1984, 201. 
gegenüber. ${ }^{13}$ Jahr für Jahr werden 300.000 mietrechtliche Prozesse geführt. ${ }^{14}$ In etwa der Hälfte der Mietverträge steht auf Vermieterseite kein privater Vermieter, sondern ein gewerbliches Unternehmen. ${ }^{15}$ Von einer persönlichen Vertrauensbeziehung zwischen den Parteien kann man daher nur in wenigen Fällen sprechen, das jeweilige moralische Risiko innerhalb der Prinzipal-Agent-Beziehung ist im Normalfall nicht abschätzbar. Daher gewinnen Sicherungsrechte erhebliche wirtschaftliche Bedeutung. Die gesetzlichen Sicherungsmöglichkeiten durch das Vermieterpfandrecht und das Zurückbehaltungsrecht erweisen sich als weitgehend wirkungslos (hierzu sogleich 1). Vertraglich vereinbarte Sicherungsrechte erscheinen auf den ersten Blick effektiver, geraten aber unvermeidlich in Konflikt mit den zwingenden mieterschützenden Vorschriften des Gesetzes (dazu unten 2).

\section{Gesetzliche Sicherungsrechte}

a) Vermieterpfandrecht

Als einziges spezifisch mietrechtliches Sicherungsmittel enthält das BGB das Vermieterpfandrecht an eingebrachten Sachen des Mieters ( $§ 562-562$ d i. V. m. 578 BGB). Seine praktische Bedeutung ist im Gegensatz zur ausführlichen gesetzlichen Regelung gering. ${ }^{16}$ Die eingebrachten Sachen des Mieters haben häufig geringen materiellen Wert, außerdem ist der Pfandverkauf umständlich. ${ }^{17}$ Ohnehin sind unpfändbare Sachen nicht erfaßt (§ 562 Abs. 1 S. 2 ZPO). Die gem. § 811 Abs. 1 Nr. 1 ZPO unpfändbaren Sachen des persönlichen Gebrauchs und des Haushalts scheiden als Sicherungsmasse von vornherein aus. Nach der herrschenden Auslegung von $\S 811$ ZPO kommen selbst Fernseher, Kühlschränke und Waschmaschinen als Pfandgegenstände nicht in Betracht. ${ }^{18}$ Soweit Hausratsgegenstände pfändbar sind, soll ihre Ver-

13 Pressemitteilung des Statistischen Bundesamts vom 30. 4. 1999, in: NZM 1999, 550; Zeimes, Die Reform des Mietrechts, 2001, 2; Ritter, Mietrecht auf dem Weg ins nächste Jahrtausend, in: NZM 1999, 146-151 (146); Lammel, Mietrechtsreform - Zurück zum BGB ?!, in: ZMR 2000, 133-139 (136). Detaillierte Auswertung für das Jahr 1987 bei Bison, Die Regulierung des Mietwohnungsmarktes in der Bundesrepublik Deutschland. Eine positive ökonomische Analyse, 1996,115.

14 Börstinghaus, Das Mietrechtsreformgesetz - Eine erste Stellungnahme aus der (gerichtlichen) Praxis, in: NZM 2000, 583-595 (583); Zeimes (wie Anm. 13), 2. - Sonnenschein, Überlegungen der Expertenkommission Wohnungspolitik zum Einfluß des Mietrechts auf den Wohnungsmarkt, in: ZMR 1996, 109-117 (110), betont, vor allem die Unberechenbarkeit der Rechtslage erhöhe die Betriebskosten des Mietrechts.

15 Allein der Gesamtverband der Wohnungswirtschaft (3.167 Mitgliedsunternehmen) und der Bundesverband freier Wohnungsunternehmen (ca. 1.700 Mitgliedsunternehmen) vermieten zusammen über 10,2 Millionen Wohnungen, Einzelheiten bei Bison (wie Anm. 13), 246.

16 Zutreffend weist Soergel/Heintzmann, Bürgerliches Gesetzbuch, 12. A. 1997, § 559 Rn. 1, darauf hin, daß die ausführliche Regelung des Selbsthilferechts die hohe Bedeutung belege, die der Gesetzgeber dem Vermieterpfandrecht eingeräumt habe.

17 Sternel, Mietrecht, 3. A. 1988, III 258; Schmidt-Futterer/Lammel, Mietrecht, 7. A. 1999, § 559 Rn. 3: Bedeutung des Pfandrechts nur noch bei Gewerberaummiete.

18 BFH NJW 1990, 1871: Farbfernseher, hierzu Lüke/Beck, Grundgesetz und Unpfändbarkeit eines Farbfernsehgeräts - BFHE 159, 421, in: JuS 1994, 22-26; LG Berlin NJW-RR 1992, 1038: Waschmaschine; Münchener Kommentar zur Zivilprozeßordnung/Schilken, 2. A. 
wertung zudem gem. $§ 812$ ZPO unterbleiben, wenn der erzielbare Erlös zum Wert der Forderung außer Verhältnis steht. Damit können etwa Möbel und Geschirr als Vollstreckungsmasse ausscheiden. ${ }^{19}$

Die Befürworter eines umfassenden Mieterschutzes bezeichnen das Vermieterpfandrecht als anachronistisch und weisen darauf hin, daß es abbedungen werden kann. ${ }^{20}$ Voelskow hält es sogar für einen "Schandfleck" des Gesetzes, daß der Vermieter berechtigt sei, die Entfernung der seinem Pfandrecht unterfallenden Sachen ohne Anrufung des Gerichts zu verhindern ( $\$ 562$ b Abs. 1 BGB $).{ }^{21}$ Auch Sternel kritisiert die Privilegierung des Selbsthilferechts gegenüber der allgemeinen Vorschrift des $\S 229$ BGB. In der Tat darf der Vermieter Selbsthilfe üben, auch wenn staatliche Hilfe rechtzeitig zu erlangen gewesen wäre. ${ }^{22}$ Die Besserstellung des Vermieters folgt jedoch aus dem unterschiedlichen Näheverhältnis der Parteien. Das allgemeine Selbsthilferecht gilt für Ansprüche aller Art, ${ }^{23}$ also auch zwischen Personen, die nur durch Zufall in rechtlichen Kontakt geraten sind. Die Parteien eines Mietverhältnisses gehen dagegen freiwillig ${ }^{24}$ eine Bindung ein. Wenn der Vermieter sein Selbsthilferecht ohne Anrufen eines Gerichts wahrnehmen kann, dient das unmittelbar der Verhinderung von Prozessen. Ohnehin greift das Selbsthilferecht nur ein, wenn der Mieter sich verdächtig verhält. Entspricht die geplante Entfernung der Sachen von dem Grundstück den gewöhnlichen Lebensverhältnissen, kann der Vermieter sein Recht nicht ausüben $\left(\S 562\right.$ a S. 2 BGB). ${ }^{25}$

Trotz der geringen praktischen Relevanz des Vermieterpfandrechts zeigt sich eine für Sicherungsmittel typische Regelung. Bei Ungewißheit über die Vertragstreue im gegenseitigen Vertrag soll das Sicherungsmittel die ordnungsgemäße Vertragsdurchführung gewährleisten, ohne zusätzliche Kosten zu verursachen. ${ }^{26}$ Das Selbsthilferecht des Vermieters ist daher mit der Verwertung einer Mietkaution zu vergleichen. In bei-

2001, § 811 Rn. 19; pfändbar dagegen sind Geschirrspülmaschinen: Baumbach/Hartmann, Zivilprozeßordnung, 60. A. 2002, § 811 Rn. 20.

19 Stein/Jonas, Kommentar zur Zivilprozeßordnung, 21. A. 1995, § 812 Rn. 1 (Münzberg), der die Vorschrift sogar extensiv auslegt; Musielak, Kommentar zur Zivilprozeßordnung, 2. A. $2000, \S 812$ Rn. 1 (Becker).

20 Sternel, Mietrecht (wie Anm. 17), III 258; Münchener Kommentar zum Bürgerlichen Gesetzbuch/Voelskow, 3. A. 1995, §559 Rn. 16; BGB-RGRK, 12. A. 1978, §559 Rn. 2 (Gelhaar).

21 MK/Voelskow (wie Anm. 20), 3. A. 1995, § 561 Rn. 2.

22 Sternel, Mietrecht (wie Anm. 17), III 268; Köhler/Kossmann, Handbuch der Wohnraummiete, 5. A. 2000, § 104 Rn. 7.

23 Palandt/Heinrichs (wie Anm. 11), § 229 Rn. 2-3.

24 Unberücksichtigt bleibt insofern die weitgehende Vererblichkeit des Mietvertrages auf Mieterseite ( $\S 563,563$ a) und der Eintritt eines Erwerbers auf der Vermieterseite ( $\$ 566)$.

25 Konkretisierung der "gewöhnlichen Lebensverhältnisse" bei Lammel, Heidelberger Kommentar zum Wohnraummietrecht, 1998, § 560 Rn. 24; Emmerich (wie Anm. 9), § 560 Rn. 11. Aus der älteren Rechtsprechung: RG Recht 1909 Nr. 1672.

26 Leider behandelt Stix, Gerichtliche und außergerichtliche Durchsetzung ziviler Rechtsansprüche. Rechtlicher Vergleich und ökonomische Analyse, 1991, nur Zivilprozesse und institutionalisierte Schiedsverfahren. Auf die Kostenersparnis eines sich aufgrund der Sicherungsmittel selbst durchsetzenden Vertrages geht sie nicht ein. 
den Fällen kann der Vermieter bei Vertragsverletzungen des Mieters seine Ansprüche sichern bzw. befriedigen, ohne gegen den Mieter zu klagen. In der Sprache der neuen Institutionenökonomik wird diese Parallelität deutlich, wenn man sowohl die vom Mieter in die Wohnung eingebrachten Gegenstände als auch eine etwaige Kautionszahlung als Geiseln bezeichnet und in diesem Druckmittel ein Indiz für einen sich selbst durchsetzenden Vertrag erblickt. ${ }^{27}$

Wegen des geringen wirtschaftlichen Werts des Vermieterpfandrechts vermag dieses Sicherungsrecht das vom Mieter ausgegehende moralische Risiko freilich nur marginal zu reduzieren. Ein Vermieter, der sich ganz auf sein Vermieterpfandrecht verlassen wollte, müßte enorme Überwachungskosten aufwenden, um ständig zu kontrollieren, welchen Wert die vom Mieter eingebrachten Gegenstände besitzen und ob dort Veränderungen eintreten. Bei Gewerbemietverträgen mag das anders sein, wenn der Mieter wertvolle Maschinen oder technische Geräte installiert. Hier erstreckt sich das Vermieterpfandrecht sogar auf das Anwartschaftsrecht, falls der Mieter seine Sachen unter Eigentumsvorbehalt erworben hat. ${ }^{28}$ Die Gewerbemiete soll aus der folgenden Betrachtung aber ausgeblendet bleiben, weil dort die privatautonome Vertragsgestaltung nicht gesetzlich beschränkt ist.

\section{b) Zurückbehaltungsrecht}

Das Zurückbehaltungsrecht ist wie das Vermieterpfandrecht ein gesetzliches Sicherungsmittel. Im Gegensatz zum Pfandrecht ist es keine mietrechtliche Besonderheit, sondern im allgemeinen Schuldrecht geregelt ( $\S 273-274,320-322$ BGB). Der Mieter kann sein Zurückbehaltungsrecht in vielfältigen Situationen ausüben. Bei Mängeln der Mietsache kann er den drei- bis fünffachen Beseitigungsaufwand zurückbehalten ${ }^{29}$. Das Zurückbehaltungsrecht ist also nicht nur Sicherungsmittel, sondern auch Druckmittel, um den Vermieter zu vertragstreuem Verhalten anzuhalten. Das Zurückbehaltungsrecht des Mieters beschränkt sich zumeist auf die Einbehaltung des Mietzinses. Gegen den Herausgabeanspruch des Vermieters kann der Mieter von Wohnraum (§ 570 BGB), Grundstücken ( 578 Abs. 1 i. V. m. § 570 BGB) und anderen Räumen (§ 578 Abs. 2 i. V. m. $§ 578$ Abs. 1 i. V. m. 570 BGB) ein Zurückbehaltungsrecht nicht geltend machen. Die gewährte Sicherheit stände in diesem Fall in keinem Verhältnis zu etwaigen Ansprüchen des Mieters. ${ }^{30}$

Schwieriger sieht es mit dem Zurückbehaltungsrecht des Vermieters aus. Nach der ursprünglichen Konzeption des BGB schied § 320 BGB von vornherein aus, da der Vermieter vorleistungspflichtig war ( 551 a. F. BGB). Die Mietrechtsreform 2001

$27 \mathrm{Zu}$ den theoretischen Hintergründen Richter/Furubotn (wie Anm. 4), 171-173.

28 Sternel, Mietrecht (wie Anm. 17), III 260.

29 Hierzu Schmidt-Futterer/Eisenschmid (wie Anm. 17), § 537 Rn. 384, der ungenau von Mietminderungsansprüchen spricht; Überblick über die Neuregelung auch bei Eisenschmid, Das Mietrechtsreformgesetz, in: WuM 2001, 215-221 (218). Zur Höhe des Zurückbehaltungsbetrages: LG Hamburg MDR 1984, 494; Joachim, Zum Leistungsverweigerungsrecht des Mieters bei Mängeln an der Mietsache, in: DB 1986, 2649-2654 (2652-2653); Palandt/Heinrichs (wie Anm. 11), § 320 Rn. 11.

30 Schmidt-Futterer/Gather (wie Anm. 17), § 556 Rn. 91. 
brachte für Wohnungen und andere Räume eine Neuregelung ( $\S 556$ b Abs 1, 579 Abs. 2 BGB). Die Miete ist jetzt zu Beginn, spätestens bis zum dritten Werktag der jeweiligen Zeitabschnitte zu zahlen. Dies führt dennoch nicht zu einer Zug-um-ZugLeistung. Eine Mietzahlung vor dem dritten Tag kann nicht verlangt werden ( $\$ 556 \mathrm{~b}$ Abs. 3 BGB), die Vorleistungspflicht des Vermieters bleibt also bestehen. Auch ein Zurückbehaltungsrecht nach § 273 BGB kommt kaum in Betracht. Wenn der Mieter eine nicht im Gegenseitigkeitsverhältnis stehende Pflicht nicht ordnungsgemäß erfüllt, z. B. eine Kaution nicht rechtzeitig entrichtet, bleibt der Vermieter trotzdem zur Überlassung verpflichtet. Eine Mietsicherheit ist frühestens zu Beginn des Mietverhältnisses fällig ( $\$ 551$ Abs. 2 S. 2 BGB), eine Vorauszahlung dürfen die Parteien nicht vereinbaren ( $\$ 551$ Abs. 4 BGB).

Angeblich soll die Umgehung dieses Verbots einfach und üblich sein. Nach Sternel machen die Vermieter die Aushändigung des Mietvertrages vom Nachweis der vollständigen Kautionszahlung abhängig. ${ }^{31}$ Viele Vermieter scheinen zum Vertragsschluß erst bereit zu sein, wenn der Mieter zuvor Sicherheit geleistet hat. In diesem Fall wären die mietrechtlichen Vorschriften nicht anwendbar, da die Mietsicherheit bereits vor Vertragsschluß zu leisten wäre. Wie verbreitet diese Praxis ist, läßt sich verläßlich nicht klären. Es zeigt sich aber ein Grundproblem zwingender Privatrechtsnormen: Wenn Sicherungsrechte und andere Vertragsgestaltungen zu stark beschnitten werden, können sich Umgehungsmöglichkeiten ergeben, die den vom Gesetz geschützten Vertragsteil noch schlechter stellen, als es ohne die Schutzvorschriften der Fall wäre. Die Einschränkung des Zurückbehaltungsrechts und die späte Fälligkeit der Mietsicherheit würden in diesem Fall dazu führen, daß Mieter, die keine Vorauszahlung leisten, überhaupt keinen Mietvertrag mehr abschließen können.

\section{Vertragliche Sicherungsrechte}

Im Gegensatz zum Vermieterpfandrecht und zum Zurückbehaltungsrecht greifen andere Sicherungsrechte nur ein, wenn die Parteien sie vereinbart haben. Speziell der Vermieter kann sein über das unergiebige Pfandrecht hinausgehendes Sicherungsinteresse nur durch vertragliche Vereinbarung befriedigen. Die volle inhaltliche Gestaltungsfreiheit besteht aber lediglich bei Mietverträgen über bewegliche Sachen, Grundstücke und Räume, die keine Wohnräume sind ( 578 Abs. 1 BGB verweist nicht auf $\S 551$ BGB). Im Wohnungsmietrecht ist der Spielraum durch zahlreiche einseitig zwingende Gesetzesnormen stark eingeschränkt.

\section{a) Vorleistungspflicht}

Das einfachste Sicherungsmittel ist die Vereinbarung einer Vorleistungspflicht. Im gegenseitigen Vertrag können die Parteien die Fälligkeit der Leistungen abweichend von $\S 271$ BGB regeln und damit $\S 320$ BGB ausschließen. ${ }^{32}$ Im Mietvertrag war früher die Vorleistungspflicht des Vermieters für die jeweiligen Zeitabschnitte der Miet-

31 Sternel, Mietrecht (wie Anm. 17), III 242.

32 Palandt/Heinrichs (wie Anm. 11), § 320 Rn. 15. 
zahlung gesetzlich fixiert, zugleich jedoch abdingbar ( $\$ 551$ a. F. BGB). Eine Vorauszahlung der Miete konnte vertraglich vereinbart werden. Die verbreitete Auffassung, diese Abweichung von $\S 551$ a. F. sei üblich gewesen, ${ }^{33}$ trifft aber nur bedingt zu. Die im Mustermietvertrag 1976 des Bundesjustizministeriums enthaltene Klausel, wonach die Miete monatlich im voraus, spätestens am dritten Werktag an den Vermieter zu zahlen war, ${ }^{34}$ schwächte die Vorleistungspflicht des Vermieters lediglich ab. Während der Mieter bis zum dritten Werktag des Monats Zeit hatte, den Mietzins zu entrichten, gewährte der Vermieter den Gebrauch der vermieteten Wohnung seit Mietbeginn im voraus. Das Sicherungsbedürfnis des Vermieters war bei einer Mietzahlung bis zum dritten Werktag eines Monats jedoch üblicherweise befriedigt. ${ }^{35}$

Inzwischen ist die bisherige Vertragspraxis zum abdingbaren gesetzlichen Leitbild erhoben worden ( $\$ 556$ b BGB) ${ }^{36}$ Rips, Eisenschmid sowie andere Autoren betonen, durch die gesetzliche Fixierung der bisherigen Vertragspraxis sei die Vorleistungspflicht des Mieters festgeschrieben worden. ${ }^{37}$ Hierauf ist genauso zu antworten wie oben: Selbst bei der erstmaligen Gebrauchsgewährung bleibt der Vermieter vorleistungspflichtig, wenn auch nur für drei Tage. In sämtlichen Folgeabschnitten der Mietzahlungsverpflichtung befindet sich der Mieter jeweils schon im Besitz seiner Wohnung, bevor er die Miete für den laufenden Monat zahlt. Aber die Sicherungskraft einer Mietvorauszahlung darf ohnehin nicht überschätzt werden. Die Miete stellt im synallagmatischen Mietvertrag lediglich das Entgelt für den Gebrauchswert der Mietsache dar, § 535 BGB. Das moralische Risiko im Prinzipal-Agent-Verhältnis zwischen Vermieter und Mieter betrifft aber nicht nur die Ungewißheit, ob der Mieter seiner Zahlungspflicht ordnungsgemäß nachkommen wird, sondern noch zahlreiche weitere Fragen wie etwa den schonenden Umgang des Mieters mit fremdem Eigentum $^{38}$ und etwaige Renovierungsleistungen beim Auszug. Diese Ungewißheiten lassen sich mit einer lediglich auf die Mietzahlung bezogenen Sicherheit nicht beseitigen, ja nicht einmal reduzieren. Selbst die Gefahr, daß der Mieter am Ende der Mietzeit seine letzten beiden Monatsraten nicht entrichtet, läßt sich durch eine Vorauszahlung kaum

33 Emmerich (wie Anm. 9), § 551 Rn. 2; Palandt/Weidenkaff (wie Anm. 11), § 556 b Rn. 1; zur älteren Praxis Palandt/Putzo, 56. A. 1997, § 551 Rn. 2.

34 Mietvertrag für Wohnräume gemäß Mustermietvertrag '76 des Bundesministers der Justiz, Ausgabe 2/91, § 3 Abs. 1; ebenso die Mietvertrags-AGB des Landesverbandes der Hessischen Haus-, Wohnungs- und Grundstückseigentümer e. V., Fassung 2/99, § 6 Nr. 1; zur zulässigen Verwendung der Klausel in AGB, die zugleich die Aufrechnungsmöglichkeiten beschränken: BGHZ 127, 245-254.

35 Zur Frage, ob sich eine entsprechende Verkehrssitte oder Gewohnheitsrecht gebildet hatte Gellwitzki, Die Mietrechtsreform - die Neuregelung zur Fälligkeit der Miete und Pacht, in: WuM 2001, 373-384 (374).

36 Referentenentwurf vom 20.3.2000, Begründung zu § 556 b Nr. 1 BGB, in: ZMR Sonderdruck Mietrechtsreformgesetz, ebenfalls in: Rips/Eisenschmid, Neues Mietrecht, 2001, 410.

37 Rips/Eisenschmid (wie Anm. 36), 81; Eisenschmid (wie Anm. 29), 217-218; ebenso unzutreffend Gellwitzki (wie Anm. 35), 376; Langenberg, Das Neue Mietrecht, in: WuM 2001, 523-532 (525).

38 In diesem Fall mag dem Vermieter ein Schadensersatzanspruch aus positiver Vertragsverletzung zustehen (ab 1. 1. $2002 \S 280$ ), der freilich oftmals nur im Prozeß durchsetzbar sein dürfte; zur Beweislastverteilung Sternel, Mietrecht (wie Anm. 17), IV 613-614. 
reduzieren, weil die Mietdauer bei unbefristeten Wohnungsmietverträgen im voraus nicht feststeht. Versucht der Vermieter jedoch, seine wirtschaftlichen Interessen, die nicht durch die Mietzahlung abgedeckt sind, zu sichern, gerät er in Konflikt mit den zwingenden Mieterschutzvorschriften.

b) Kaution

Das Gesetz benutzt den Begriff Kaution nicht, sondern spricht in $\S 551$ BGB von Mietsicherheiten. ${ }^{39} \mathrm{Ob}$ die Parteien eine Mietsicherheit vereinbaren und in welcher Form sie zu leisten ist, unterliegt ihrer inhaltlichen Gestaltungsfreiheit. ${ }^{40}$ Durch das 1983 in Kraft getretene "Gesetz zur Erhöhung des Angebots an Mietwohnungen" wurde die Freiheit der Parteien beschränkt und eine Sicherheitshöchstgrenze von drei Monatsmieten eingeführt. Im Interesse des Mieterschutzes ist diese Vorschrift einseitig unabdingbar ( $\$ 551$ Abs. 4 BGB). Neben der Höchstsummenbegrenzung greifen weitere zwingende Vorschriften ein. Der Mieter ist berechtigt, die Kaution in drei gleichen monatlichen Teilzahlungen zu leisten. Die erste Rate ist frühestens zu Beginn des Mietverhältnisses fällig, § 551 Abs. 2 BGB. Damit ist nicht der Vertragsschluß, sondern der Zeitpunkt der Überlassung gemeint. ${ }^{41}$ Eine Vertragsklausel, die den Mieter zu einer Einmalzahlung verpflichtet, ist demnach nichtig. ${ }^{42}$ Zahlt der Mieter die Kaution nicht, darf der Vermieter aus diesem Grunde nicht fristlos kündigen, ${ }^{43}$ sondern kann lediglich Zahlungsklage erheben. Diese Klage ist selbst nach der Beendigung des Mietverhältnisses noch möglich, wenn der Vermieter sein fortdauerndes Sicherungsinteresse darlegt. ${ }^{44}$

Wegen der zwingenden Obergrenze für die Kaution und der ebenfalls unabdingbaren Teilleistungsberechtigung des Mieters ist die inhaltliche Gestaltungsfreiheit erheblich beschränkt. Obwohl das vom Mieter ausgehende moralische Risiko im Einzelfall unterschiedlich hoch sein kann, erlaubt das Gesetz keine Abstufungen über die Dreimonatshöhe hinaus. Die summenmäßig reduzierte Sicherheit vermag das moralische Risiko somit zwar zu reduzieren, nicht jedoch auf Null. Die Überwachungskosten des Vermieters werden mithin nicht überflüssig, da die Kaution einen im Vergleich zum Wert der gemieteten Wohnung oder gar eines Hauses geringen Wert nicht überschreiten darf. Der Vermieter muß also weiterhin um den pfleglichen Umgang mit seinem Eigentum besorgt sein. Außerdem kann eine auf drei Monatsmieten beschränkte Kaution gerade in einem Konflikt zwischen Mieter und Vermieter ihren wirtschaftlichen Wert dadurch einbüßen, daß der Mieter vor seinem Auszug die letzten beiden Monatsmieten nicht bezahlt. Das außerordentliche Kündigungsrecht des Vermieters gem. § 543 Abs. 2 Nr. 3. a) BGB läuft in diesem Fall leer. Der Vermieter kann sich

39 Die rechtlichen Konstruktionsmöglichkeiten sind verschieden: Sternel, Mietrecht aktuell, 3. A. 1996, Rn. 1333.

40 Sternel, Mietrecht (wie Anm. 17), III 221; Emmerich (wie Anm. 9), § 550 b Rn. 2.

41 LG Mannheim ZMR 1990, 18-19; Sternel, Mietrecht aktuell (wie Anm. 39), A 1335; Staudinger/Emmerich (wie Anm. 8), § 550 b Rn. 17.

42 LG München I WuM 2001, 280-281.

43 Wetekamp, Kommentar zum BGB-Mietrecht, 1998, § 550 b Rn. 21.

44 LG Düsseldorf WuM 2001, 487-488. 
zwar aus der Kaution befriedigen, verliert aber dann zugleich seine Sicherheit für die übrigen vom Mieter ausgehenden moralischen Risiken.

Es liegt nahe, an dieser Stelle unmittelbar nach den Folgen der rechtlichen Ungleichheit zu fragen und zu prüfen, ob und auf welche Weise ein Vermieter seine Sicherungsinteressen in erlaubten Vertragsklauseln durchsetzen kann. Zuvor jedoch ist das Problem noch weiter zuzuspitzen. Es zeigt sich nämlich, daß auch die übrigen denkbaren Sicherungsrechte die Transaktionskosten des Vermieters nicht so stark senken, daß das aufgrund des Wohnungsbesitzes des Mieters typische Ungleichgewicht des Prinzipal-Agenten-Verhältnisses behoben wäre.

\section{c) Bürgschaft}

Auch eine Mietbürgschaft des Mieters oder eines Dritten stellt eine Mietsicherheit gem. $§ 551$ Abs. 1 BGB dar. Die Bürgschaft darf sich damit wie sämtliche Mietsicherheiten höchstens auf den Betrag von drei Nettomieten beziehen. ${ }^{45}$ Lediglich in Ausnahmefällen läßt die Rechtsprechung Abweichungen zu, und zwar immer dann, wenn ein Dritter freiwillig eine Bürgschaft unter der Bedingung übernimmt, daß ein Wohnungsmietvertrag zustandekommt. ${ }^{46}$ Auch eine Bürgschaft, die eine fristlose Kündigung wegen Zahlungsverzugs abwenden oder den Vermieter zur Rücknahme einer bereits ausgesprochenen Kündigung bewegen soll, darf eine höhere Sicherheit gewähren. ${ }^{47}$ Entscheidend ist, daß der Vermieter in beiden Fällen keinen Anspruch auf die höhere Sicherheit besitzt, sondern der Bürge sie ohne rechtlichen oder wirtschaftlichen Druck von sich aus gewährt.

Rechtlich erfüllt eine Mietbürgschaft dieselbe Sicherungsfunktion wie eine Kautionszahlung. Wirtschaftlich ist sie jedoch grundverschieden. Die Kaution stammt aus dem Vermögen des Mieters und stellt im Rahmen des Prinzipal-Agent-Verhältnisses eine sog. Geisel dar, da der Mieter ein eigenes Interesse daran besitzt, die Kaution am Ende der Mietzeit wieder ausbezahlt zu erhalten. Eine Beschränkung auf drei Monatsmieten läßt sich hier mit dem Argument begründen, finanzschwache Mieter hätten sonst nicht die Möglichkeit, die Mietsicherheit aufzubringen. Eine Mietbürgschaft wirft dagegen andere Fragen auf. Das Vermögen des Mieters bleibt bei dieser Sicherung unangetastet. Da eine Bankbürgschaft wegen der hohen Avalprovision bei unbefristeten Mietverträgen wirtschaftlich unattraktiv ist, ${ }^{48}$ wird der Mieter im Regelfall

45 BGHZ 107, 210 (Leitsatz); ausführliche Begründung bei Sternel, Mietrecht (wie Anm. 17), III $257 \mathrm{c}$.

46 BGHZ 111, 361 (Leitsatz); zustimmend Palandt/Weidenkaff (wie Anm. 11), § 551 Rn. 3; ablehnend Tiedtke, Die Auswirkungen des $\S 551$ BGB auf die Bürgschaft eines Dritten für Mietsicherheiten, in: ZMR 1990, 401-404; kritisch auch Sternel, Mietrecht aktuell (wie Anm. 39), Rn. 1363; zweifelnd Staudinger/Emmerich (wie Anm. 8), § 550 b Rn. 11; Soergel/Heintzmann (wie Anm. 16), § 550 b Rn. 4, erweitert diese Regel auf sämtliche Arten freiwillig gewährter Sicherheiten.

47 LG Kiel NJW-RR 1991, 1291-1292; zustimmend Palandt/Weidenkaff (wie Anm. 11), § 551 Rn. 3.

48 Valentin, Die Mietsicherheitshöchstgrenze des $\$ 550$ b Abs. 1 Satz 1 BGB, in: ZMR 1992, 1-5 (2), hält dagegen die an die Bank zu zahlende Bereitstellungsgebühr für wirtschaftlich unerheblich. Lützenkirchen, Die Entwicklung des Mietrechts in der obergerichtlichen 
eine ihm nahestehende Person aus seiner Verwandtschaft oder seinem Freundeskreis bitten, die Bürgschaft zu übernehmen. Das ist vor allem bei der Vermietung von Wohnraum an Studenten verbreitet. Hier übernehmen häufig die Eltern eine Mietbürgschaft. ${ }^{49}$ Das Prinzipal-Agent-Verhältnis zwischen Vermieter und Mieter verwandelt sich damit in eine Dreipersonenbeziehung. Der Mieter wird nunmehr auch zum Agenten des Bürgen, da er zusätzlich dafür verantwortlich ist, daß der Bürge nicht vom Vermieter in Anspruch genommen wird. Jetzt sind also zwei Prinzipale, nämlich Vermieter und Bürge, am vertragsgerechten Verhalten des Mieters interessiert. Für den Vermieter bedeutet das eine erhebliche Reduktion seiner Transaktionskosten, denn ein Teil der Überwachung des Mieters übernimmt jetzt der Bürge aus eigenem wirtschaftlichen Interesse. Bei einer engen emotionalen Bindung zwischen dem Bürgen und dem Mieter bedeutet das für den Mieter einen hohen Anreiz zu vertragsgemäßem Verhalten. Das für Prinzipal-Agent-Verhältnisse typische moralische Risiko wird durch eine Mietbürgschaft somit erheblich stärker verringert als durch eine Kautionszahlung.

Für den Vermieter ergeben sich dennoch zwei Probleme: Zum einen wird die Bürgschaft im Gegensatz zu einer Spareinlage nicht verzinst. Die Höhe der Sicherheitsleistung reduziert sich somit entsprechend dem Geldwertverfall, und parallel zum sinkenden Wert der Sicherheit erhöht sich im Laufe der Mietdauer das moralische Risiko. ${ }^{50}$ Zum anderen ist eine Mietbürgschaft im Vergleich zur Kaution für den Vermieter mit zusätzlichen Transaktionskosten verbunden. Falls er den Bürgen aus der Bürgschaft in Anspruch nehmen möchte, trägt er dessen Liquiditätsrisiko und muß sich gegebenenfalls mit einem wirtschaftlich wertlosen Vollstreckungstitel begnügen. Weshalb die Höhe einer Mietbürgschaft auf drei Monatsmieten begrenzt ist, läßt sich nur schwer beantworten. Der BGH rechtfertigt die Höchstgrenze mit der Überlegung, anderenfalls werde die wirtschaftliche Bewegungsfreiheit des Mieters zu stark eingeschränkt. ${ }^{51}$ Dieses Argument hält die Rechtsprechung jedoch nicht konsequent durch, denn eine freiwillig, unabhängig von einer Aufforderung durch den Vermieter übernommene Bürgschaft eines Dritten darf durchaus einen höheren Betrag umfassen, obwohl der rechtliche und wirtschaftliche Druck auf den Mieter wegen der Rückgriffsmöglichkeit des Bürgen gem. § 774 Abs. 1 S. 1 BGB derselbe bleibt. Erstaunlicherweise scheinen empirische Erhebungen zur Beeinträchtigung der Bewegungsfreiheit des Mieters bei Bürgschaftsübernahmen zu fehlen. Je nach rechtspolitischen Vorlieben lassen sich damit entweder sämtliche Mietbürgschaften als Belastungen des Mieters ansehen ${ }^{52}$ oder aber als überhaupt nicht belastend bewerten. ${ }^{53}$ Im Gegensatz zur Rechtfertigung einer Höchstsumme für Kautionszahlungen läßt sich

Rechtsprechung des Jahres 2000, in: WuM 2001, 55-71 (61), vermutet, daß Bankbürgschaften zunehmende Bedeutung im Wohnungsmietrecht erlangen werden.

49 Schmidt-Futterer/Blank (wie Anm. 17), § 550 b Rn. 11.

50 Zur gewöhnlichen Geldentwertung als Vermieterrisiko Bub/Treier, Handbuch der Geschäfts- und Wohnraummiete, 3. A. 1999, II 635 (Bub).

51 BGHZ 107, 210 (213).

52 Tiedtke (wie Anm. 46), 403.

53 Valentin (wie Anm. 48), 2-3. 
eine Begrenzung der Mietbürgschaften sowohl juristisch-dogmatisch als auch empirisch-ökonomisch schwer begründen.

\section{d) Vertragsstrafe}

Eine Vertragsstrafe stellt eines der wirkungsvollsten zivilrechtlichen Druckmittel dar. Sie soll Vertragstreue des Schuldners dadurch erreichen, daß ihm bei Verstoß gegen seine Pflichten ein erheblicher Geldverlust droht. ${ }^{54}$ Der Gläubiger braucht die Nichterfüllung der schuldnerischen Verpflichtungen nicht zu beweisen ( $\$ 345$ BGB), und selbst der Nachweis eines entstandenen Schadens ist ihm erspart. ${ }^{55}$

In Mietverträgen über Wohnraum ist eine Abrede, durch die sich der Vermieter vom Mieter eine Vertragsstrafe versprechen läßt, seit 1964 unwirksam ( $\$ 555$ BGB). ${ }^{56}$ Das Verbot soll verhindern, daß sich der Vermieter unangemessene Druckmittel zur Durchsetzung seiner Rechte gewähren läßt. ${ }^{57}$ Verstöße führen freilich entgegen $§ 139$ BGB nicht zur Nichtigkeit des gesamten Mietvertrages,${ }^{58}$ sondern lediglich zur Nichtigkeit der verbotenen Klausel. Das Verbot von Vertragsstrafen wird umfassend ausgelegt. Auch Verwirkungs- und Verfallsklauseln, durch die etwa die Rückzahlung einer Kaution bei vorzeitiger Aufhebung eines Mietvertrages ausgeschlossen wird, sind unwirksam. ${ }^{59}$ Streitig ist die Behandlung von Schadensersatzpauschalen. Der Wortlaut der Norm spricht klar gegen die Gleichsetzung, auch wird dem Mieter bei pauschaliertem Schadensersatz der Einwand, es sei gar kein Schaden entstanden, nicht abgeschnitten ( $\$ 309$ Nr. 5 BGB). In der Rechtsprechung sind pauschalierte Mahngebühren und andere Kosten dagegen mehrfach für unzulässig gehalten worden. ${ }^{60}$ Eine Regelung, die die inhaltliche Gestaltungsfreiheit der Parteien beschränkt, nämlich das

54 Zum Sicherungszweck BGHZ 49, 84 (89); 63, 256 (259); Larenz, Schuldrecht I, 14. A. 1987, 376.

55 BGHZ 63, 256 (259): erleichterte Schadloshaltung; BGHZ 105, 24 (27): erleichterte Schadloshaltung ohne Einzelnachweis; zustimmend Palandt/Heinrichs (wie Anm. 11), Rn. 1 vor $\S \S 339-345$; zur Beweislast bei $\S 345$ auch Larenz (wie Anm. 54), 381.

$56 \S 550$ a a. F.: BGBl. I, S. 1912; zur Entstehungsgeschichte Schmidt-Futterer/Blank (wie Anm. 17), § 550 a Rn. 1.

57 Rips/Eisenschmid (wie Anm. 36), 76; Blank/Börstinghaus, Miete. BGB-Mietrecht und MHG, 2000, § 550 a Rn. 1; Schmidt-Futterer/Blank (wie Anm. 17), § 550 a Rn. 1; andere Rechtfertigung des Gesetzeszwecks unabhängig von Verteilungsproblemen bei Lammel, Heidelberger Kommentar (wie Anm. 25), § 550 a Rn. 1-4: Vermeidung von Störungen in der Entwicklung der Partnerschaft zwischen den Parteien sowie Verhinderung der Aushöhlung des Mieterschutzes bei einer vom Vermieter angestrebten Vertragsbeendigung; ohne nähere Begründung Soergel/Heintzmann (wie Anm. 16): Die Regelung bezwecke den Schutz den Mieters.

58 Sternel, Mietrecht (wie Anm. 17), III 292.

59 Sternel, Mietrecht (wie Anm. 17), III 288. Zur Gleichsetzung einer Verfallklausel mit einer Vertragsstrafe BGH NJW 1960, 1568; Palandt/Weidenkaff (wie Anm. 11), § 555 Rn. 1; Schmidt-Futterer/Blank (wie Anm. 17), § 550 a Rn. 4.

60 AG Freiburg WuM 1986, 368; AG Darmstadt WuM 1988, 109; OLG Hamburg ZMR 1990, 270: zulässige Pauschalkosten für vorzeitige Vertragsaufhebung; Sternel, Mietrecht (wie Anm. 17), III 289. Abweichende Entscheidungen zu pauschalen Weitervermietungskosten: LG Lübeck WuM 1985, 114; AG Hamburg WuM 1985, 113. 
Verbot der Vertragsstrafen im Wohnungsmietrecht, wird somit extensiv ausgelegt. Der Grundsatz, daß Ausnahmevorschriften nicht ausgedehnt werden dürfen, wird im Wohnungsmietrecht offenbar nur bedingt anerkannt. Wegen des marktbedingten Ungleichgewichts zwischen Mietern und Vermietern und der hohen Bedeutung der Wohnung als Sozialgut ist die extensive Auslegung des Vertragsstrafeverbots weitgehend anerkannt. ${ }^{61}$

Ökonomisch ist die Vertragsstrafe ein typisches Sicherungsmittel, um den ex-postOpportunismus einer Partei, das sog. moralische Risiko, zu reduzieren. Die Beweislastumkehr erleichtert dem Vermieter die Prozeßführung und senkt auf diese Weise die mit der Rechtsverfolgung verbundenen Transaktionskosten. Die Notwendigkeit der gerichtlichen Geltendmachung bedeutet für den Vermieter jedoch eine Erschwerung gegenüber einer Kautionsleistung. Die Verwertung einer Kaution mag in der Tat leichter sein als die Eintreibung einer Vertragsstrafe. Andererseits kann der Schuldner zum Zeitpunkt des Vertragsschlusses der Vertragsstrafe womöglich leichter zustimmen als einer Sicherheitsleistung, da sein Vermögen unmittelbar nicht beeinträchtigt wird. Die Vertragsstrafe stellt den Schulder daher nicht ohne weiteres schlechter als eine Kautionszahlung. Auch im Vergleich zur Mietbürgschaft wird der Mieter nicht zwangsläufig stärker belastet. Der Verfall der Vertragsstrafe mag wirtschaftlich mit dem Regreß des Bürgen vergleichbar sein. Entscheidend ist jedoch, daß die Vertragsstafe weiterhin ein Zweipersonenverhältnis betrifft und daher auf den Mieter kein Druck von Seiten eines Dritten ausgeübt werden kann.

Das für die Kautionsleistung gängige Argument, die wirtschaftliche Bewegungsfreiheit des Mieters dürfe nicht übermäßig eingeengt werden, trifft das Problem von Vertragsstrafen offenkundig nicht. Hier geht es lediglich darum, ein vertragsgemäßes Verhalten durch die ständige Strafdrohung zu erzwingen. Das Verbot von Vertragsstrafen verringert somit den Anreiz des Mieters, sich vertragsgerecht zu verhalten, und erhöht damit das moralische Risiko des ex-post-Opportunismus. Möglicherweise wollte der Gesetzgeber der Gefahr vorbeugen, daß ein Vermieter mit Aussicht auf eine großzügige Vertragsstrafe gar kein Interesse an einem vertragsgemäßem Verhalten des Mieters hat, wenn die Einziehung der Strafe wirtschaftlich attraktiver als die Vertragserfüllung ist. ${ }^{62}$ Dieses Risiko ist freilich wegen der dem Schuldner gem. § 343 BGB unabdingbar eingeräumten Möglichkeit, die Herabsetzung einer unverhältnismäßig hohen Strafe zu verlangen, begrenzt. ${ }^{63}$ Das Verbot von Vertragsstrafen im Wohnungsmietrecht führt damit zum Ausschluß eines Sicherungsmittels, das in anderen Bereichen des Geschäftsverkehrs sehr wirkungsvoll eingesetzt wird. ${ }^{64}$

61 Sternel, Mietrecht (wie Anm. 17), I 272; Ausdehnung auf Umgehungsgeschäfte befürwortet Staudinger/Emmerich (wie Anm. 8), § 550 a Rn. 5.

$62 \mathrm{Zu}$ dieser Gefahr Eger (wie Anm. 7), 188, Lösungsmöglichkeit ebd. 182: Die gewährte Sicherheit hat für den Agenten hohe, für den Prinzipal dagegen geringen Wert; zu den Gefahren der Vertragsstrafe auch Lammel, Heidelberger Kommentar (wie Anm. 25), § 550 a Rn. 1.

63 Zur Unabdingbarkeit Palandt/Heinrichs (wie Anm. 11), § 343 Rn. 2.

64 Beispiele bei Eger (wie Anm. 7), 189-191; BGHZ 85, 305-315 (308): "Derartige Klauseln sind im Baugewerbe üblich." - Zur Vertragsstrafe bei Geschäftsraummiete und Pacht Bub (wie Anm. 50), II 530; Schmidt-Futterer/Blank (wie Anm. 17), § 550 a Rn. 10. 


\section{e) Weitere Sicherungsmittel}

Mit Kaution, Bürgschaft und der verbotenen Vertragsstrafe sind die drei prinzipiell verschiedenen Sicherungsrechte erfaßt. Entweder stellt der Schuldner eine Kaution, um das moralische Risiko seines ex-post-Opportunismus zu verringern, oder er erhöht durch eine Bürgenbestellung den moralischen Druck auf sich selbst. Die übrigen im Wohnungsmietrecht denkbaren und zulässigen Sicherungsmittel lassen sich zwanglos einem dieser Modelle zuordnen. Sowohl die Hinterlegung, Sicherungsabtretung als auch die Sicherungsübereignung sind wirtschaftlich mit einer Kautionsleistung vergleichbar. ${ }^{65}$ Lediglich eine Mietsicherungsdienstbarkeit fällt aus zwei Gründen aus dem Rahmen. Zum einen ist sie lediglich bei langfristigen Gewerbemietverträgen gebräuchlich, zum anderen stellt sie ein ausschließlich zugunsten des Mieters wirkendes dingliches Sicherungsrecht dar. ${ }^{66}$ Die prinzipielle Ähnlichkeit der Sicherungsmittel findet ihre gesetzliche Bestätigung darin, daß auch hier die unabdingbare Höchstgrenze von drei Monatsmieten nicht überstiegen werden darf, § 551 Abs. 1 BGB. ${ }^{67}$ Hierbei ist der Wert mehrerer Sicherungsmittel zusammenzurechnen. ${ }^{68}$ Damit gerät nun die Frage ins Blickfeld, welche Auswirkungen die gesetzliche Beschränkung der Sicherungsmöglichkeiten entfaltet.

\section{Die Auswirkungen der beschränkten Sicherungsmöglichkeiten auf die Vertragsgestaltung}

Auf den ersten Blick erreichen die Höchstsummenbegrenzung der Mietsicherheiten und das Verbot von Vertragsstrafen zwei vom Gesetzgeber verfolgte Zwecke. Zum einen bleiben dem Mieter zu Beginn eines Mietverhältnisses übermäßige wirtschaftliche Belastungen erspart. Zum anderen können auch wirtschaftlich schwache Mietinteressenten mit zahlungskräftigen Mitbewerbern auf dem Wohnungsmarkt konkurrieren. In Anlehnung an Valentin kann man von einem Belastungs- und einem Wettbewerbsargument sprechen. ${ }^{69}$ Diesen aus dem Blickwinkel des Mieters positiven Folgen stehen mindestens drei Nachteile gegenüber, die die vom Gesetzgeber beabsichtigten Wirkungen relativieren, wenn nicht sogar aufheben. Es sind dies die Ungeeignetheit der Regelung für zahlreiche Sicherungsmittel, die pauschale Benachteiligung bestimmter Mietergruppen am Wohnungsmarkt sowie die Einpreisung der Sicherungshöhe in den Mietzins.

65 Überblick über weitere, in der Praxis nicht so bedeutende Sicherungsmittel bei Emmerich (wie Anm. 9), § 550 b Rn. 2; Schmidt-Futterer/Blank (wie Anm. 17), § 550 b Rn. 17; einen Sonderfall beschreibt Uellenberg, Mietausfall-Versicherung mit Arbeitslosen-Zusatzversicherung, in: WuM 1998, 263-265; zur Abtretung von Arbeitseinkommen BAG WuM 2001, 116-119, sowie Derleder/Stapelfeld, Die Lohn und Gehaltsabtretung als Vermietersicherung, in: ZMR 1987, 123-128.

66 Umfassend Stiegele, Die Mietsicherungsdienstbarkeit - ein fiduziarisches Sicherungsrecht zur dinglichen Sicherung von Mietverhältnissen, 1995.

67 Anwendbarkeit des $\S 550$ b a. F. BGB ( $\$ 551$ n. F. BGB) auf sämtliche Mietsicherheiten in BGHZ 107, 210.

68 BGHZ 107, 210 (212); Emmerich (wie Anm. 9), § 550 b Rn. 3; Bub (wie Anm. 50), II 445.

69 Valentin (wie Anm. 48), 2. 
Das Belastungsargument kann die gesetzliche Regelung nur bedingt rechtfertigen. Der Schutz des Mieters vor einer übermäßigen Kautionszahlung bewahrt den durch Umzug und Maklercourtage ohnehin finanziell belasteten Wohnungsmieter in der Tat vor übermäßigen Beeinträchtigungen. Im Falle einer Mietbürgschaft gilt diese Erwägung aber nicht. Auch andere Sicherungsmittel, die wie etwa eine Sicherungsübereignung wirtschaftlich weiterhin dem Mieter verbleiben, lassen sich mit dem Belastungsargument nicht erfassen. Geht man davon aus, daß die Privatautonomie über Art. 2 Abs. 1 GG Verfassungsrang besitzt, so verstößt die einschränkungslose Höchstsummenbegrenzung der Sicherheiten gegen den Verhältnismäßigkeitsgrundsatz, da die Ausdehnung des zwingenden Rechts auf Bürgschaften und Sicherungsübereignungen gar nicht geeignet ist, den vom Gesetzgeber verfolgten Zweck zu erreichen. ${ }^{70}$ Daher mag man daran denken, § 551 Abs. 1 BGB verfassungskonform lediglich auf diejenigen Sicherheiten anzuwenden, die den Mieter zum Zeitpunkt der Bestellung wirtschaftlich belasten. Hierbei handelt es sich freilich um rechtliche Konsequenzen, die die gesetzliche Regelung nicht grundsätzlich in Frage stellen. Erheblich gravierender sind die vom Gesetzgeber unbeabsichtigten wirtschaftlichen Folgen der Regelung.

So führt die Höchstsummenbegrenzung der Mietsicherheiten mit hoher Wahrscheinlichkeit zur kollektiven Benachteiligung potentieller Risikogruppen am Wohnungsmarkt. Als Risikogruppen werden diejenigen Mieter verstanden, die aufgrund ihrer sozialen Herkunft ein höheres moralisches Risiko verkörpern als ein Durchschnittsmieter. Geht man davon aus, daß Vermieter und Mieter sich nicht kennen und eine Sicherheitsleistung in Höhe von drei Monatsmieten das durchschnittliche wirtschaftliche Gegengewicht zum ex-post-Opportunismus des Mieters bildet, ändert sich dieses Gleichgewicht, wenn Mieter und Vermieter einander bekannt sind und das moralische Risiko genauer einschätzen können. Im Falle einer Vertrauensbeziehung zwischen Freunden und Bekannten mögen die Risiken gering sein, so daß der Vermieter sich mit einer geringeren Kautionszahlung zufriedengeben kann. ${ }^{71}$ Kennt der Vermieter dagegen positiv die Unzuverlässigkeit des Wohnungsinteressenten, wird er nicht bereit sein, an ihn zu vermieten. Problematisch sind diejenigen Fälle, in denen die Parteien sich nicht persönlich kennen, dem Vermieter aber wenigstens eine Eigenschaft des Mieters bekannt ist, die ihn einer Risikogruppe zuordnet. Hier ist vor allem an sozialschwache kinderreiche Familien, an Ausländer und an Wohngemeinschaften zu denken. Im Einzelfall können diese Mieter den Vertrag genauso zuverlässig erfüllen wie kinderlose Doppelverdiener. Viele Vermieter befürchten jedoch, daß es in diesen Fällen überdurchschnittlich häufig zu Schwierigkeiten kommt. ${ }^{72}$ Für das Prinzipal-Agent-

70 Zutreffend gesehen von Valentin (wie Anm. 48), 5.

71 Gegenüberstellung eines Tauschverhältnisses unter Bekannten und unter Fremden im Hinblick auf Vertragstreue und Transaktionskosten bei North, Institutionen, institutioneller Wandel und Wirtschaftsleistung, 1992, 66.

72 Die Rechtsprechung weist gelegentlich darauf hin, daß keine systematischen empirischen Untersuchungen existieren, die die These erhärten, eine Vermietung an Ausländer oder Wohngemeinschaften sei für den Vermieter mit Nachteilen verbunden: OLG Stuttgart WuM 1982, 129 (130); OLG Hamm WuM 1983, 108 (110). Immerhin gaben aber in einer 1997 durchgeführten Umfrage 66 \% der Vermieter an, sie vermieteten nur an die aus ihrer 
Verhältnis bedeutet das, daß der Vermieter das generell höhere moralische Risiko bei einer Vermietung an Ausländer oder andere Randgruppen nur dann eingehen wird, wenn er eine höhere Sicherheit als beim Durchschnittsmieter erhält. Das ist gesetzlich aber verboten, so daß das Gleichgewicht zwischen Risiko und Sicherheit nicht mehr besteht. Dadurch sinkt die Bereitschaft zur Wohnungsvermietung an Randgruppen, der entsprechende Teilmarkt wird künstlich verknappt. Das OLG Hamm bemerkte hierzu 1986 mit einer erstaunlichen Nüchternheit: "Daß sich solche Verknappungen regelmäßig gerade gegenüber sozial schwächergestellten Mieterschichten ergeben, liegt von vornherein auf der Hand." ${ }^{, 73}$ Bereits vier Jahre zuvor stellte Eike von Hippel fest, daß die mieterschützende Gesetzgebung ganz offensichtlich zu erheblichen Störungen des Wohnungsmarks und zur Entstehung einer neuen Wohnungsnot für sozial Schwache geführt habe. ${ }^{74}$ Derartige Überlegungen mögen politisch nicht korrekt wirken und unsozial erscheinen. Diesem Einwand ist jedoch bereits Friedrich August von Hayek in seiner Untersuchung über den Mieterschutz aus dem Jahre 1928 entgegengetreten. Danach ist es erstens methodisch unzulässig, die Ergebnisse wissenschaftlicher Untersuchungen deswegen zu bestreiten, weil sie herzlos wirken. Zweitens ist es im Ergebnis noch unverantwortlicher, bestimmte tatsächliche Zusammenhänge zu ignorieren, als die unerwünschten Nebenwirkungen sozialpolitischer Maßnahmen offenzulegen. Denn nur eine Bestandsaufnahme der rechtlichen und wirtschaftlichen Verhältnisse kann den Mietern, in deren Interesse das soziale Mietrecht geschaffen wurde, wirklich helfen. ${ }^{75}$

Freilich gibt es für Vermieter einen Ausweg, das von bestimmten Mietergruppen ausgehende moralische Risiko trotz einer gesetzlichen Höchstsummenbeschränkung der Mietsicherheiten auf sich zu nehmen. Die Lösung besteht darin, das Mietausfallrisiko sowie die Gefahren von Beschädigungen der Wohnung und mangelhafter Renovierung von vornherein in einen höheren Mietzins einzukalkulieren. ${ }^{76}$ Der Teilmarkt für benachteiligte Mietergruppen ist somit nicht nur verknappt, sondern auch überteuert. Die Miete stellt in diesen Fällen nicht nur das Entgelt für die Gebrauchsüberlassung dar, sondern enthält zugleich einen Risikozuschlag, der die Problemgruppen finanziell belastet. Im Gegensatz zu einer Sicherheitsleistung ist dies ein doppelter Nachteil. Zum einen nimmt bei einer längeren Mietdauer der überhöhte Mietpreis den Sicherungswert einer vielfachen Monatsmiete an, und zum zweiten wird er im Gegensatz zu einer Kaution nicht zurückgezahlt. Damit steht der Mieter aufgrund des gesetzlichen Mieterschutzes wirtschaftlich erheblich schlechter, als wenn er den Risikozuschlag in Form einer höheren Kaution zahlen müßte. Da die Miethöhe zum Zeitpunkt des Vertrags-

Sicht besten Mieter: Veser/Jaedicke, Analyse der Auswirkungen des Mietrechts und Bewertung des Änderungsbedarfs, in: WuM 1997, 197-202 (197).

73 OLG Hamm WuM 1986, 206 (207); ähnlich Sonnenschein (wie Anm. 14), 110.

74 v. Hippel, Der Schutz des Schwächeren, 1982, 21.

75 Hayek, Das Mieterschutzproblem (1928), jetzt in: ders., Wirtschaft, Wissenschaft und Politik. Aufsätze zur Wirtschaftspolitik, 2001, 181-207 (207).

76 Allgemein hierzu Lammel, Heidelberger Kommentar (wie Anm. 25), § 559 Rn. 3; Bub/ Treier/Schultz (wie Anm. 50), III 263; vor diesem Hintergrund jetzt auch BVerfG WuM 2001, 540-541. 
schlusses der inhaltlichen Gestaltungsfreiheit der Parteien unterliegt, greifen die mieterschützenden zwingenden Rechtsnormen in diesem Fall nicht ein.

In der Praxis bleibt potentiellen Problemmietern oft nichts anderes übrig, als minderwertige unrenovierte Altbauwohnungen zu überteuerten Preisen zu mieten. ${ }^{77}$ Selbst dies wird aber durch die gesetzliche Konzeption erschwert. Das Gesetz macht dem Vermieter die Renovierung von Altbauten nämlich dadurch attraktiv, daß es ihm ein besonderes Recht zur Mieterhöhung bei Modernisierungen einräumt, §559 Abs. 1 BGB. Damit wird der für soziale Randgruppen zur Verfügung stehende Wohnraum zusätzlich verknappt, was sich wiederum in steigenden Mieten auswirkt. Dieses Problem kann die Rechtsprechung allein nicht lösen. Zwar haben mehrere Oberlandesgerichte entschieden, daß ein Risikozuschlag für Ausländer und andere Randgruppen nicht zu einem eigenen Teilmarkt mit von vornherein höheren Vergleichsmieten führen darf. ${ }^{78}$ Jedoch wirken sich die höheren Quadratmeterpreise durchaus auf den allgemeinen Mietspiegel aus. Die Mietpreise für die Wohnungen benachteiligter Mietergruppen dürfen daher bis zu $20 \%$ über der ohnehin erhöhten Vergleichsmiete liegen. ${ }^{79}$

Die Wohnraumverknappung für Risikogruppen und damit die Benachteiligung sozial schwacher Mietinteressenten, verbunden mit einem überhöhten Mietzins sind unbeabsichtigte Nebenfolgen der zwingenden Mieterschutzvorschriften. Das geltende Mietrecht führt damit zu Ergebnissen, die den vom Gesetzgeber verfolgten Zielen geradewegs entgegenlaufen. $\mathrm{Ob}$ in der Gesamtbilanz die rechtliche und wirtschaftliche Situation der Mieter tatsächlich verbessert wurde, läßt sich durchaus bezweifeln. ${ }^{80} \mathrm{Bei}$ diesem Befund handelt es sich um keinen Einzelfall. Ausgehend von diesem mietrechtlichen Beispiel soll im folgenden gezeigt werden, daß die zum "Schutz" beschlossenen Eingriffe des Staates in das Privatrecht fast immer ungewollte Nebenwirkungen entfalten und damit als Regelungsmittel sehr zurückhaltend beurteilt werden müssen.

\section{Das Grundproblem: Gesetzgeberische Eingriffe in die Privatautonomie und ihre Folgen}

Die unbeabsichtigten Auswirkungen des gesetzlichen Mieterschutzes lenken den Blick auf ein seit langem bekanntes Grundproblem. Staatliche Planung und Regulierung ganzer Lebensbereiche sind nur beschränkt möglich. Der Grund hierfür liegt in der Komplexität der Sozialordnung, ökonomisch ausgedrückt in der asymmetrischen Information. Die neue Institutionenökonomik hat herausgearbeitet, daß niemand, der

77 Ipsen, Wohnungen und Mieten, Analysen zur Auswirkung des Wohnraumkündigungsschutzgesetzes, in: Archiv für Kommunalwissenschaften 1976, 262-279 (271): Die empirische Untersuchung für das Jahr 1975 ergab, daß Ausländer für einfache bzw. schlechte Wohnungen zwischen $39 \%$ und $45 \%$ höhere Mieten zahlen als Deutsche.

78 OLG Stuttgart WuM 1982, 129-131; OLG Hamm WuM 1983, 108-112; OLG Hamm WuM 1986, 206-207.

79 Neue Obergrenze für Mietpreisüberhöhungen gem. § 5 Wirtschaftsstrafgesetz 1954 i. d. F. von Art. 5 Mietrechtsreformgesetz vom 19. 6. 2001.

80 So bereits vor einem Vierteljahrhundert Weitnauer, Der Schutz des Schwächeren im Zivilrecht, 1975, 34. 
einen Vertrag schließt, sämtliche für seine Entscheidung wichtigen Tatsachen bis in alle Einzelheiten kennt. Der Aufwand für die Informationsbeschaffung wäre beispielsweise zu hoch, wollte man sämtliche Angebote einholen, um sich dann für das preiswerteste $\mathrm{zu}$ entscheiden. Der Informationsgrad der Menschen ist somit unterschiedlich. Das gilt auch für die Teilnehmer am Rechtsverkehr, etwa die Wohnungssuchenden und die Hauseigentümer. Trotz ihrer unterschiedlichen Interessen gelingt es ihnen aber, für beide Seiten vorteilhafte Verträge zu schließen. Wenn der Staat hierfür den Rechtsrahmen zur Verfügung stellt, erleichtert er den Rechtsverkehr und senkt im besten Fall sogar die Transaktionskosten, wenn das dispositive Recht den Parteien die mühsame Aushandlung der Einzelheiten abnimmt. Die Vorverlagerung der Fälligkeit der Mietzahlung im Rahmen der Mietrechtsreform hat das Recht an die seit langem bestehende Verkehrssitte angenähert und damit die Vertragsverhandlungen vereinfacht. Eine derartige Rechtsänderung verfolgt freilich nicht das Ziel, das Sozialleben zu planen oder zu ändern.

Ein anderes Bild ergibt sich, wenn der Staat ganze Rechtsbereiche wie das Wohnungsmietrecht weitgehend aus der Privatautonomie herausnimmt und durch zwingende Rechtsnormen regelt. Da ein staatlicher Gesetzgeber in keinem Fall sämtliche verstreuten, wenn auch unvollständigen Informationen der einzelnen Teilnehmer am Rechtsverkehr sammeln kann, trifft auch er seine gesetzgeberischen Entscheidungen notwendigerweise vor dem Hintergrund unvollständiger Information. Welche Auswirkungen einzelne staatliche Maßnahmen haben, ist daher oftmals nicht abzusehen, weil die Ausweich- und Umgehungsmöglichkeiten der Marktteilnehmer nicht vollständig vorausgesehen werden können. ${ }^{81}$ Die Ausweichmöglichkeiten sind die zwar nicht voraussehbare, aber unvermeidliche Folge der für die privatautonome Vertragsgestaltung verbleibenden Spielräume. Wenn bestimmte Einzelentscheidungen durch gesetzgeberische Eingriffe aus der Dispositionsbefugnis der Parteien herausgenommen werden, werden diese versuchen, ihre Interessen an anderer Stelle durchzusetzen. Eine gesetzgeberische Einschränkung der Vertragsfreiheit wird daher immer zur Folge haben, daß die rechtlich benachteiligte Partei sich zumindest wirtschaftlich schadlos hält. ${ }^{82}$ Eidenmüller hat dieses Problem an einem fiktiven Mietrechtsfall, einer gesetzlich zwingenden Mindestausstattung von Wohnräumen, untersucht und kommt zu einem gleichlautenden Ergebnis. Die Präzision des Zivilrechts als Umverteilungsinstrument ist danach nicht nur zweifelhaft, sondern gerade Feinsteuerungen dürften nur schwer möglich sein. ${ }^{83}$

81 Solche gesetzlichen Maßnahmen sind sogar verfassungsrechtlich fragwürdig, da die Voraussehbarkeit gesetzgeberischer Maßnahmen ein rechtsstaatliches Gebot darstellt: BVerfGE 7, 89 (92); 25, 269 (290); hierzu Weitnauer (wie Anm. 80), 29.

82 Ähnlich Honsell (wie Anm. 8), 140: Ein überzogenes ius cogens verursacht Detailregelungen und eine ausufernde Kasuistik, die ihrerseits die Gesetzesumgehung begünstigen.

83 Eidenmüller, Effizienz als Rechtsprinzip. Möglichkeiten und Grenzen der ökonomischen Analyse des Rechts, 2. A. 1998, 296-306; im Ergebnis ähnlich Honsell (wie Anm. 8), 153, die in der gesetzlichen Annäherung an öffentlichrechtliche Inpflichtnahmen eine Gefahr für das Prinzip der Privatautonomie sehen. 
Diese Konsequenz ist aus der Sicht des planenden Gesetzgebers besonders ärgerlich, denn die Einschränkung der Privatautonomie erfolgt ja gerade deswegen, um eine unerfahrene oder wirtschaftlich benachteiligte Partei im Rechtsverkehr zu schützen. Genau diese Personen können sich aufgrund ihrer tatsächlich schwächeren Position gegen die neuen Vertragsbedingungen, die die andere Partei durchsetzen möchte, kaum zur Wehr setzen. Wenn das zwingende Recht Ungleichgewichte in bestimmten Bereichen beseitigt, schafft es damit nicht die Grundlage für die effektive Wahrnehmung der Privatautonomie durch beide Parteien. Selbst wenn die Gleichstellung und damit ausgewogene Verhandlungsbedingungen in bestimmen Bereichen verwirklicht werden, entstehen sofort neue Schieflagen. Versucht man diese wiederum durch neue zwingende Rechtsnormen auszugleichen, so entsteht ein staatsinterventionistischer Teufelskreis, der erst dann zum Abschluß gelangt, wenn das privatrechtliche Verhalten der Einzelpersonen dem sozialpolitischen Leitbild des Gesetzgebers entspricht. $\mathrm{Da}$ es sich bei diesen Erwägungen nicht um unbegründete Befürchtungen handelt, belegt ein kurzer Blick in das Änderungsregister zum BGB. In den ersten 90 Jahren seines Bestehens, von 1896 bis 1985, wurde das Gesetzbuch 84 mal geändert. Von 1986 bis 1990 ergingen 19 Änderungen, von 1991 bis 1995 folgten bereits 22 Änderungen und von 1996 bis 2000 waren es schließlich 27 Änderungsgesetze, die das bürgerliche Recht umgestalteten. Allein 2001 kam es zu 11 Änderungsgesetzen. ${ }^{84}$ Die gesetzgeberischen Eingriffe entfalten offenkundig eine sich selbst beschleunigende Eigendynamik. ${ }^{85}$

Der Ausweg aus der Umwandlung des Privatrechts in staatliches Planungsrecht ist ebenso verblüffend einfach wie politisch schwer durchsetzbar. Es spricht viel dafür, daß eine Rückkehr zur Vertragsfreiheit zahlreiche Verteilungsprobleme und Ungleichgewichte beseitigen würde. ${ }^{86}$ Die bisherigen Ausweich- und Umgehungsmöglichkeiten, die der wirtschaftlich stärkeren Partei zur Verfügung stehen, wären dann überflüssig und würden aus der Vertragspraxis mit hoher Wahrscheinlichkeit verschwinden. Da die Zahl der Wohnungen wie auch die Zahl der Mietinteressenten sich durch die Gesetzgebung nicht ändern, ist die Gefahr starker Mietsteigerungen bei einer Freigabe des Wohnungsmarks eher gering. Außerdem sind selbst unter Geltung des sozialen Mietrechts die Mietpreise für Neubauten im Erstbezug in München zwischen September 2000 und September 2001 um über $16 \%$ gestiegen. ${ }^{87}$

84 Aus Vereinfachungsgründen wurde - wie etwa im Schönfelder üblich - lediglich die Zahl der ändernden Gesetze erfaßt.

85 Deutliche Kritik am zunehmenden Staatsinterventionismus im Wohnungsmietrecht bei Staudinger/Emmerich (wie Anm. 8), vor $\S 535,536$ Rn. 27-29; ähnlich Lammel, Nochmals: Mietrechtsreform - Systematik und Dogmatik, in: ZMR 2000, 808-811 (809). Bison (wie Anm. 13), 233, führt die Regulierungsspirale im Mietrecht vor allem auf die politische Macht der Mieter als Wählerpotential zurück.

86 Kronberger Kreis, Mehr Mut zum Markt - Konkrete Problemlösungen, 1986, 20; MK/ Voelskow (wie Anm. 20), Rn. 59 vor § 535, mit der Erwägung, eine Verschärfung des Mieterschutzes hemme Investitionen und verzögere damit die Überwindung von Schwierigkeiten auf dem Wohnungsmarkt.

87 Pressemitteilung des Ringes deutscher Makler, in: NZM 2001, Heft 20, V-VI. 
Die Argumente gegen eine Stärkung der Vertragsfreiheit im Mietrecht haben unterschiedliches Gewicht. Köhler/Kossmann betonen, die Gesetze der Marktwirtschaft könnten im Wohnungsmietrecht nicht uneingeschränkt gelten, da die Ware Grundstück nicht beliebig vermehrbar sei. ${ }^{88}$ Diese Erwägung überzeugt nicht. Auch Aktien sind nicht beliebig vermehrbar ebenso wie Gemälde berühmter Maler. Dennoch entsprechen Börsenhandel und Kunstauktionen geradezu klassischen marktwirtschaftlichen Modellen. ${ }^{89}$ Auch die verbreitete Behauptung, wegen der grundlegenden Bedeutung einer Wohnung für menschenwürdiges Leben seien Einschränkungen der Privatautonomie erforderlich, trifft nicht den Kern des Problems. Denn ebenso wie das Dach über dem Kopf gehören auch Nahrung, Kleidung und Heizung zu menschlichen Grundbedürfnissen. Wieso der Staat die Kompetenz besitzen soll, durch zwingendes Recht eine Wertigkeit von Bedürfnissen festzulegen, ist kaum nachvollziehbar. ${ }^{90}$ Der Hinweis, in einem Sozialstaat sei die Wohnung eben kein Wirtschafts-, sondern ein Sozialgut, ${ }^{11}$ besitzt keine Substanz, denn mit dem Wort "sozial” lassen sich sämtliche wünschenswerten Lebensverhältnisse begründen, ohne daß es möglich wäre, über die Einzelheiten je Einigkeit zu erzielen. ${ }^{92}$

Gewichtiger ist demgegenüber die Lehre von der Materialisierung der Privatautonomie bzw. vom emanzipierenden Schutz. Nach dieser Auffassung sind Einschränkungen der formalen Vertragsfreiheit notwendig, um ein in der Realität nicht bestehendes Gleichgewicht auf gesetzlichen Wege herzustellen. Die Beschränkung der Privatautonomie verfolgt nach Sternel den Zweck, die "intellektuelle und wirtschaftliche Unfähigkeit (...) breiter Bevölkerungsschichten"93 auszugleichen und sie in die Lage zu versetzen, aktiv die Vertragsbedingungen auszuhandeln. Eleganter hat Drexl eine staatliche Pflicht hergeleitet, die Voraussetzungen für das Funktionieren der auf freien Entschlüssen der Marktteilnehmer basierenden Privatautonomie aktiv zu gestalten. ${ }^{94}$ Auch Honsell, der sich für eine weitgehende Rückkehr zur Vertragsfreiheit im Wohnungsmietrecht ausspricht, begrüßt zwingende Gesetzesnormen zum Schutz des schwächeren Vertragspartners wie das Verbot von Vertragsstrafen und die Pflicht zur Verzinsung der Kaution. ${ }^{95}$

88 Köhler/Kossmann (wie Anm. 22), 6.

89 Richter/Furubotn (wie Anm. 4), 315, 329, mit dem Hinweis, daß es sich bei der Börse um einen streng geschlossenen Markt mit Zugangsbeschränkungen handele.

90 Kronberger Kreis (wie Anm. 12), 183-184.

91 Sternel, Mietrecht (wie Anm. 17), I 271.

92 Hayek, Der Atavismus "sozialer Gerechtigkeit” (1976); Die Illusion sozialer Gerechtigkeit (1976), beides in: Die Anmaßung von Wissen, 1996, 181-203; ders., Die verhängnisvolle Anmaßung. Die Irrtümer des Sozialismus, 1996, 124-129.

93 Sternel, Mietrecht (wie Anm. 17), I 270 Anm. 3. Ob die Unterstellung, breite Bevölkerungsschichten seien wirtschaftlich und intellektuell unfähig, nicht ebenso "inhuman" ist, wie Sternel die Befürworter der Vertragsfreiheit tituliert, braucht an dieser Stelle nicht entschieden zu werden.

94 Drexl, Die wirtschaftliche Selbstbestimmung des Verbrauchers. Eine Studie zum Privatund Wirtschaftsrecht unter Berücksichtigung gemeinschaftsrechtlicher Bezüge, 1998, 258.

95 Honsell (wie Anm. 8), 164, 186. 
Die Analyse der ökonomischen Auswirkungen des mieterschützenden zwingenden Gesetzesrechts hat die Nachteile gesetzgeberischer Eingriffe klar gezeigt. Ob die Beschränkung der mietrechtlichen Sicherungsmittel die Wohnungssuche für potentielle Risikogruppen wirklich erleichtert, läßt sich bezweifeln. Daher gebührt der verfassungsrechtlichen Entscheidung zugunsten der Privatautonomie der Vorrang, selbst wenn es sich bei der Vertragsfreiheit nur um ein Auffanggrundrecht handelt. ${ }^{96}$ Angesichts der sich stetig beschleunigenden Gesetzesänderungen und der dennoch bzw. gerade deswegen neu entstehenden Ungleichgewichte stützt die Untersuchung der Sicherungsrechte im Mietvertrag die Forderung nach der Entstaatlichung des Mietrechts und nach mehr Mut zum Markt. ${ }^{97}$

\section{Zusammenfassung}

Die vorliegende Untersuchung beschäftigt sich mit Sicherungsmitteln des Vermieters im Wohnungsmietrecht. Die Themenbeschränkung ermöglicht es, die Auswirkungen staatlicher Beschränkungen der Privatautonomie sehr genau nachzuzeichnen. Es zeigt sich, daß das im Gesetz vorgesehene Vermieterpfandrecht kaum Bedeutung besitzt. Auch das Zurückbehaltungsrecht nützt dem Vermieter nichts, da er trotz der Vorverlegung der Mietzahlungsfälligkeit weiterhin vorleistungspflichtig bleibt. Eine Absicherung durch Vertragsstrafen scheidet ebenfalls aus, da diese im Wohnungsmietrecht verboten sind. Die größte Bedeutung besitzen vertraglich vereinbarte Mietsicherheiten wie Kaution und Mietbürgschaft. Diese sind der Höhe nach auf drei Monatsmieten begrenzt. Der Gesetzgeber wollte damit außerordentliche Belastungen des Mieters zum Zeitpunkt des Wohnungswechsels vermeiden und wirtschaftlich schwächeren Bevölkerungskreisen die Teilnahme am Wohnungsmarkt ermöglichen. Die Beschränkung der Vertragsfreiheit führt jedoch dazu, daß bei bestimmten Mietergruppen wie Ausländern und Wohngemeinschaften ein Ungleichgewicht zwischen dem unkalkulierbaren moralischen Risiko des ex-post-Opportunismus und der beschränkten Mietsicherheit besteht. Die Vermieter lösen diese Schwierigkeit durch Verknappung des Wohnungsangebots für Problemgruppen und Risikoaufschläge auf den Mietzins. So stehen sich gerade diejenigen Gruppen wirtschaftlich schlechter, zu deren Schutz die Höchstummenbegrenzung eingeführt worden ist.

Dieses Ergebnis ist die unvermeidbare Folge staatlicher Lenkungsversuche im Privatrecht. Die partielle Beschränkung der Privatautonomie führt immer dazu, daß die überlegene Partei Ausweichmöglichkeiten findet und ihre Interessen in denjenigen Punkten durchsetzt, für die die Vertragsfreiheit fortbesteht. Im Mietrecht ist dies vor allem die Miethöhe, und so ist die Vermietung überteuerter und qualitativ schlechter Wohnungen an wirtschaftlich schlechtergestellte Bevölkerungsgruppen eine unbeab-

96 Drexl (wie Anm. 94), 257, der Eingriffe in die Privatautonomie als ultima ratio bezeichnet.

97 Kronberger Kreis, Konkrete Problemlösungen (wie Anm. 86), 20. - v. Stebut, Der soziale Schutz als Regelungsproblem des Vertragsrechts, 1982, zeigt am Beispiel der Kündigungsschutzregeln, daß dem Mieter die zwingenden Normen in der Realität gar nicht helfen. 
sichtigte Nebenfolge des staatlichen Mieterschutzes. Eine Rückkehr zur Vertragsfreiheit im Mietrecht käme daher gerade denjenigen Personen zugute, zu deren Schutz die Privatautonomie beschränkt wurde. Hier auf Änderungen zu hoffen, ist freilich unrealistisch. Schon 1958 zeigte Günter Dürig am Beispiel des Mietrechts, daß staatliche Beschränkungsnormen "geradezu naturgesetzlich" zu ihrer eigenen Aufrechterhaltung tendieren und der Sozialstaat seine einmal gewonnenen Gestaltungsspielräume kaum wieder aufgibt. Dabei ist es der Staat, der seine den Bürgern abgerungenen Handlungen stets neu vor der Verfassung legitimieren muß. ${ }^{98}$

So bleibt der Markt aus der Wohnungswirtschaft weitgehend ausgeklammert. Die dadurch verfestigten Schieflagen werden weiterhin staatliche Eingriffe und die Schaffung neuer zwingender Normen rechtfertigen. ${ }^{99}$ Demgegenüber muß die Zivilrechtswissenschaft unbeirrt auf Privatrechtsprinzipien verweisen und die Tauglichkeit der Vertragsfreiheit für Problemlösungen hervorheben. Diese Aufgabe ist nicht populär. Sie ist aber notwendig, um das Verständnis für die Voraussetzungen eines funktionierenden Privatrechts zu schärfen.
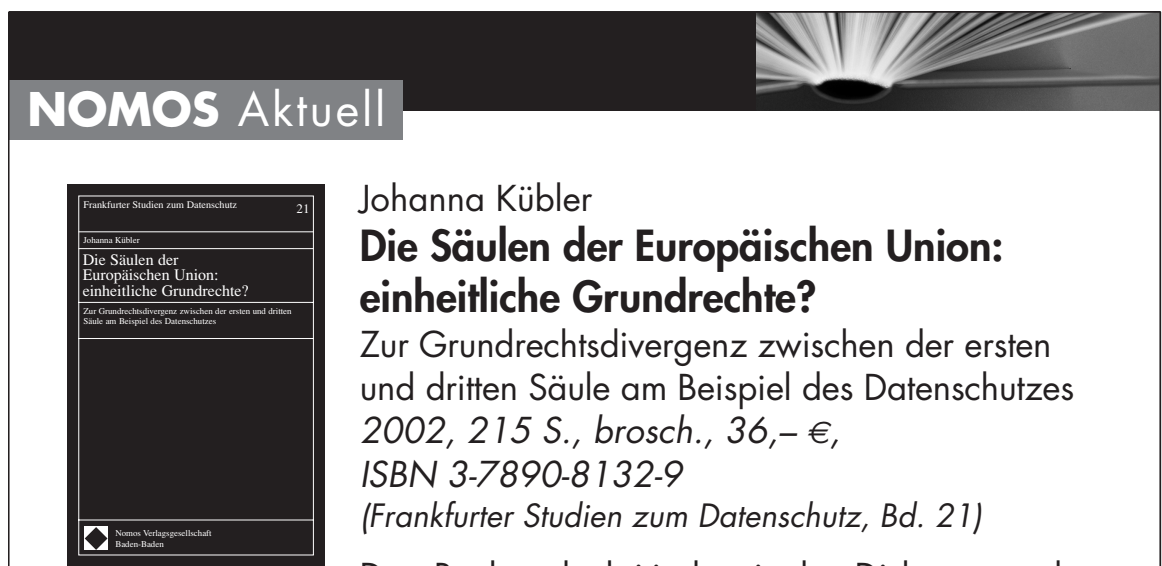

Johanna Kübler

Die Säulen der Europäischen Union: einheitliche Grundrechte?

Zur Grundrechtsdivergenz zwischen der ersten und dritten Säule am Beispiel des Datenschutzes 2002, 215 S., brosch., 36,- $€$, ISBN 3-7890-8132-9

(Frankfurter Studien zum Datenschutz, Bd. 21)

Das Buch geht kritisch mit der Diskrepanz des Grundrechtsschutzes innerhalb der Europäischen Union um. Es zeigt am Beispiel der Datenschutzrichtlinie und der Europol-Konvention diese Diskrepanzen auf und erarbeitet Vorschläge für eine säulenübergreifende Regelung.

NOMOS Verlagsgesellschaft • Baden-Baden

Baden-Baden · Fax 07221/2104-43 ·nomos@nomos.de

98 Maunz/Dürig, Grundgesetz-Kommentar, Art. 2 Abs. 1 Rn. 61 (Stand 1958); pessimistische Einschätzung auch von Hayek, Wohin zielt die Demokratie? (1976), in: ders., Die AnmaBung (wie Anm. 92), 204-215.

99 Bison (wie Anm. 13), 264-265, begründet die Regulierungsintensität überzeugend mit der Dominanz der Mieter in der Wählerschaft und meint, langfristig könne nur eine erhebliche Erhöhung der Wohneigentumsquote zu einer Liberalisierung des Mietrechts führen. 